\title{
On the Nature of Dark Matter and Dark Energy
}

\author{
Baurov Yury Alexeevich ${ }^{1}$, Malov Igor Fedorovich ${ }^{2}$ \\ ${ }^{1}$ Central Research Institute of Machine Building, Korolyov, Russia \\ ${ }^{2}$ P. N. Lebedev Physical Institute, Russian Academy of Sciences, Moscow, Russia \\ E-mail:baurov@mail.ru,malov@prao.ru \\ Received February 19, 2010; revised March 17, 2010; accepted March 20, 2010
}

\begin{abstract}
It is known that all candidates in dark matter (DM) particles (neutrinos, axions, supersymmetric particles etc.) can not explain the basic properties of DM. The same can be said on the proposed candidates in dark energy (DE) (for example, quintessence). In the paper it is shown that some problems connected with DM and DE can be solved in the framework of the byuon theory. Basic axioms and some conclusions of this theory are discussed. The existence of fundamental unobserved elements in nature, byuons is declared. Physical space in our Universe is the quantum medium of special objects $4 \mathrm{~b}$, formed in four-contact interactions of byuons $\left(\mathrm{m}_{4 \mathrm{~b}} \mathrm{c}^{2} \approx 33 \mathrm{eV}\right)$. These objects determine the average density of substance (DM) in the Universe $\sim 10^{-29} \mathrm{~g}$ $\mathrm{cm}^{-3}$. The byuon theory predicts a new interaction of natural objects with physical vacuum. This new force can cause the observed acceleration of our Universe. The estimations show that it is higher than the gravitational force at distances of order to $10^{26}-10^{28} \mathrm{~cm}$. Some other consequences of the byuon theory are considered.
\end{abstract}

Keywords: Dark Matter, Dark Energy, Byuon, New Force

\section{Introduction}

Some hard problems have appeared in astrophysics during the last dozens of years. Observations show that approximately $4 \%$ of the cosmological energy density is accounted for by baryons, $23 \%$ by "the dark matter" and the reminder by "the dark energy" (see, for example, $[1,2])$.

There are some evidences for the existence of dark matter (DM) and dark energy (DE). Here we enumerate basic ones only.

1) In 1937 F.Zwicky measured velocities of galaxies in the Coma cluster and concluded that the total mass of this cluster must be much more than observable one to prevent the escaping of investigated galaxies from the cluster.

2) The summarized mass of the observed gas and galaxies in the number of clusters is not enough to keep them inside of the cluster.

3) The gravitational lensing by clusters of galaxies gives the mass of such lens much more than calculated masses of clusters.

4) The rotation curves of galaxies [3] show that the total mass of the individual galaxy is approximately one order higher than the mass of gas and all stars observed in this galaxy.
5) The observations of supernovae in distant galaxies (see, for example, [4]) show that our Universe expanses with an acceleration, and there is a source causing such type of expansion.

The nature of dark matter and dark energy is unknown up to now.

\section{Dark Matter}

DM is not observed as a shining matter and must be characterized by extremely weak electromagnetic interactions. It must be approximately collisionless and nonrelativistic.

DM is not primarily baryonic. The calculated amount of deuterium should be much smaller than observed one if the average baryon density was an order of magnitude higher than the modern value ( $\sim 0.3$ baryons per cubic meter).

The mass interval for the possible candidates in DM is huge (from $10^{-22} \mathrm{eV}$ to $10^{6} \mathrm{M}_{\odot} \approx 10^{72} \mathrm{eV}$ ).

Let us discuss the most probable candidates in DM.

1) Axions, light pseudo-scalar bosons $[5,6]$ with mass $\mu \mathrm{eV} \lesssim \mathrm{m} \lesssim \mathrm{meV}$. They could be detected by resonant axion-photon conversion in a magnetic field $[7,8]$.

2) Neutrinos. Some laboratory experiments and cos- 
mological restrictions give the mass interval for all kinds of neutrinos:

$50 \mathrm{meV} \lesssim \Sigma \mathrm{m}_{v} \lesssim 0.7 \mathrm{eV}$, or $0.0005<\Omega_{v} \mathrm{~h}^{2}<0.0076$, where $\Omega_{v}=\rho_{v} / \rho_{\mathrm{c}}, \mathrm{h}=\mathrm{H}_{0} / 100 \mathrm{~km} / \mathrm{sec} / \mathrm{Mpc}, \rho_{\mathrm{c}}=3 \mathrm{H}_{0}{ }^{2} /$ $(8 \pi \mathrm{G})$ is the critical density of the Universe, $\mathrm{H}_{0}$ is the Hubble constant.

Super-symmetric theories put bosons and fermions in common multipletes. They give some possible candidates in DM.

1) The super-partner of the graviton, gravitino with the spin $3 / 2$ [9].

2) Neutralinos. These are the four spin $1 / 2$ Majorana fermion super-partners of the neutral gauge and Higgs bosons $\left(\chi_{1-4}^{0}\right)$ [10]. There are also two charged Dirac fermion super-partners of charge gauge and Higgs bosons, charginos $\left(\chi_{1-2}^{ \pm}\right)$.

3) Axinos, a spin $1 / 2$ partner of the axion [11].

4) Non-topological solitons, Q-balls [12].

5) If our four-dimensional space-time is embedded in a higher dimensional space, the Kaluza - Klein excitations of Standard Model states along the orthogonal dimensions may be as DM candidates [13].

6) Objects of many dimensions (branes) are described in string theories. Their fluctuations have been considered as particles (branons) which could be DM candidates [14].

7) DM could be an ordinary matter in the mirror world where the only communication is gravitational. In this case our Universe and a mirror universe are two branes in a higher dimensional space [15].

8) At the last stages of inflation gravitational interactions can produce a lot of weakly interacting massive particles which for mass scales of $10^{13} \mathrm{GeV}$ could account for DM [16].

9) Primordial black holes have been considered as candidates in DM as well [17].

So, as observations give, baryons provide approximately $4 \%$ of DM, neutrinos $\sim 0.3-3 \%$ of it. The rest $(20-25 \%)$ is a non-baryonic DM. The nature of this part of DM is unclear. There are many problems with theoretical foundations and experimental evidences of the existence of particles mentioned above and described in cited papers.

\section{Dark Energy}

The nature of DE is much more unclear than that of DM. It is necessary for it to have the equation of state of the following form (see, for example, [18]):

$$
\mathrm{p}=\mathrm{w} \rho
$$

where $p$ is pressure and $\rho$ is the energy density. The most probable value of the parameter $\mathrm{w}$ is approximately -1 , as follows from the known observations. This implies that the energy density of such substance is constant and corresponds to the flat universe, i.e. the curvature $\mathrm{K}$ of the spatial sections (slices at constant cosmic time) is equal to zero. DE causes the acceleration of the expansion of our Universe. Figure 1 shows the sum (solid line) of two potentials: The usual (negative) gravitational potential $\varphi_{1}$ (broken line) causing the attraction of two bodies and a positive constant potential $\varphi_{2}$ giving the repulsion at large distances $\left(r>r_{*}\right)$.

One of the possible sources of DE is "quintessence" [19], a scalar field $\Phi$ rolling slowly in a potential. Most quintessence models give for such scalar fields

$$
\mathrm{m}_{\Phi} \mathrm{c}^{2} \sim 10^{-33} \mathrm{eV}
$$

In quantum field theory light scalar fields are hard to understand. In any case these fields should give rise to long-range forces which must be observable. It is surprisingly why such quintessence field has not been detected up to now.

There are many problems with other models of DE (see, for example, [18]).

In this paper we shall try to explain DM and DE in the framework of the byuon theory. First of all we will describe briefly the foundations of this theory.

\section{Basic Axioms and Hypotheses: Space, Time, and Physical Vacuum in the Light of the Byuon Theory}

Any theory begins with axioms, that is, with basic postulates accepted without proofs.

Thus, let us assume that there are no space, no time, no world of elementary particles of which all physical bodies consist, but there is an unobservable object, a byuon $\Phi(i)$ [20-25], being unobservable in itself and characterized by discrete states (i.e. numbered by the series of natural numbers) having inherent "vectorial" property. The expression for $\Phi(i)$ is

$$
\boldsymbol{\Phi}(i)=\left[\mathbf{A}_{\mathbf{g}} X(i)\right] \vee\left(-\sqrt{-1} \quad\left[\mathbf{A}_{\mathbf{g}} X(i)\right]\right)
$$

where $X(i)$ is "length" of the byuon, a real (positive or negative) value depending on the index $i=0,1,2, \ldots \mathrm{k}, \ldots$.

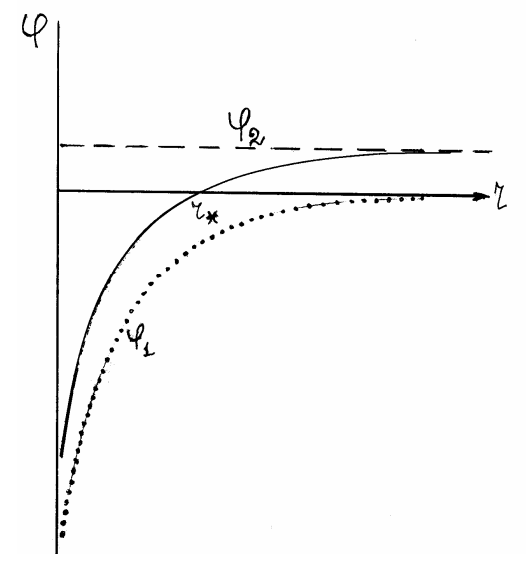

Figure 1. Scheme of two body interactions in our Universe. 
Index $i$ is a quantum number for $\Phi(i)^{1}$. The explanation of square brackets has been given further. The dimension of byuons is equal to the dimension of electric charge (in the CGSE-system) or of magnetic flux or of the Dirac's monopole. The quantity $\mathrm{A}_{\mathrm{g}}$ is some internal potential being equal in magnitude to the cosmological vectorial potential $\mathbf{A}_{\mathrm{g}}$, a new fundamental vectorial constant introduced in $[26,27]\left(\mathrm{A}_{\mathrm{g}} \approx 1.95 \cdot 10^{11} \mathrm{Gs} \cdot \mathrm{cm}\right)$.

Thus, $\vec{\Phi}(i)$ can take both real and pure imaginary values.

The whole set $\Phi(i)$ forms a one-dimensional space $R_{1}$ in index $i$.

According to this conception, by the discrete time is meant, for the byuon, a discrete change in the index $i$ (its increase or decrease) is possible. In connection with the discrete time, a quantum of time $\tau_{0}$ and quantum of space $\tilde{x}_{0}$ are introduced in the one-dimensional discrete space $R_{1}$ formed by byuons ( $\tau_{0} \approx 0.9 \times 10^{-43} \mathrm{~s}, \tilde{x}_{0} \approx 2.8 \times 10^{-33}$ $\mathrm{cm})$. The distance between byuons is defined therewith as a difference in their lengths $x(i)$. The space $R_{1}$ is discrete by definition.

Since the space $R_{1}$ is discrete, one of methods of parametrization of $\mathrm{X}(i)$ is $\mathrm{X}(i)=\tilde{x}_{o} \cdot i$, or $\mathrm{X}(i)=-\tilde{x}_{o} \cdot i$.

Statics. In the set $\{\Phi(i)\}$, there are meant no static states with time $\mathrm{t}>\tau_{0}$.

Kinematics. Depending on whether the length $X(i)$ positive or negative, decreases or increases in magnitude, free byuons (i.e. not interacting one with another) can be only in one of the four so called vacuum states (VS) $I I^{+}$, $I^{+}, I, I I$. Further we will omit sometimes VS in the expressions like VS $I I^{+}$.

Introduce the following definitions.

1) A free byuon is in the state $I^{+}$if its positive length discretely, in a quantum of time $\tau_{0}$, increases by a quantum of distance $\tilde{x}_{0}$ with the speed of propagation (increase in length) $c=\frac{\widetilde{x}_{0}-0}{\tau_{0}}=c_{0} \quad\left(c_{0}\right.$ is the light speed $)$.

Hence the speed of byuons is the ratio of their lengths to the postulated quantum of time.

2) A free byuon is in the state $I^{+}$if its positive length discretely, in a quantum of time $\tau_{0}$, decreases by $\tilde{x}_{0}$. In this case $c=\frac{0-\tilde{x}_{0}}{\tau_{0}}=-c_{0}$.

3) A free byuon is in $I \Gamma$ if the modulus of its negative length increases by $\tilde{x}_{0}$ in time $\tau_{0}$ with $c=\frac{-\tilde{x}_{0}-0}{\tau_{0}}$ $=-c_{0}$.

4) A free byuon is in $I$ if the modulus of its negative

${ }^{1}$ It should be explained that the vector $\Phi(i)$ is not an ordinary vector in some space but an object with "inner" vectorial properties that are manifesting themselves when the value $x(i)$ changes in the process of physical space formation. length discretely, in time $\tau_{0}$, decreases by $\tilde{x}_{0}$. In this case $c=\frac{0-\left(-\widetilde{x}_{0}\right)}{\tau_{0}}=c_{0}$.

From the definition of byuons it is seen that they are in perpetual dynamics of generation and annihilation, extension and contraction. The collection of free (not interacting) byuons in VSs $I I^{+}, I^{+}, I, I \Gamma$ forms physical vacuum of the one-dimensional space $R_{1}$ of index $i$ (about properties of $R_{1}$ will be said below). Recall however that in this model of physical vacuum, time is a sequence of events of byuon generation (extension) and "collapse" (contraction). These correspond to each byuon its own count of time measured by the natural number series. One of the two directions of the one-dimensional space $R_{1}$, coincident with that of a byuon with the maximum $x(i)$ in $\mathrm{VS} I I^{+}$, is taken for the positive direction of the vector $\vec{A}_{\mathrm{G}}$ and $\vec{\Phi}(i)$.

The average magnitudes for byuons being in the above described VSs at maximum $i=k$, are determined from the following expressions (see 1, and [20-22]):

$$
\begin{array}{ll}
{[\vec{A} \cdot X]_{I I^{+}}^{i+1}=\vec{A}_{G}\left[\frac{2 i+1}{2} \cdot \frac{\text { const }_{1}}{k \cdot A_{G}}\right]} & X>0, \\
{[\vec{A} \cdot X]_{I^{+}}^{i}=-\sqrt{-1} \cdot \vec{A}_{G}\left[\frac{2 i+1}{2} \cdot \frac{\text { const }}{k \cdot A_{G}}\right]} & X>0, \\
{[\vec{A} \cdot X]_{I I^{-}}^{k-i}=\sqrt{-1} \cdot \vec{A}_{G}\left[\frac{2(k-i)-1}{2} \cdot \frac{\text { const }_{1}}{k \cdot A_{G}}\right]} & X<0, \\
{[\vec{A} \cdot X]_{I^{-}}^{k-i-1}=-\vec{A}_{G}\left[\frac{2(k-i)-1}{2} \cdot \frac{\text { const }}{k \cdot A_{G}}\right]} & X<0,
\end{array}
$$

where const $_{1}=\frac{\sqrt{h c_{0}}}{4 \sqrt{3}} \cdot \frac{h c_{0}}{e_{0}^{2}}$. is some constant. As we will see later $\mathrm{h}$ is equal to the Plank constant and $\mathrm{e}_{0}$ is the electron charge. (See Appendix 1). The square brackets mean the average value for byuons between previous and subsequent magnitudes because any observations are possible during time intervals much more than a time quantum only.

Assume that for the byuons with the length greater than $\tilde{x}_{0}$, only contact interactions are realized, by which we will mean existence of at least two byuon VSs at a quantum of space $R_{1}$.

Hypothesis 1. Assume the observable three-Dimensional space $R_{3}$ to appear as a result of minimization of the potential energy (PE) of VSs byuon interactions in the one-dimensional space $R_{1}$ formed by them. We construct PE from the taking into consideration of dimensions. More precisely, the space $R_{3}$ is fixed by us as the result of this byuon dynamics. In the space $R_{3}$ therewith the dynamical processes for objects with the residual positive potential energy of byuon interactions originate, and in consequence, the wave properties of elementary 
particles arise.

PE means the extreme value of the expression with the dimension of energy. This expression is formed using all possible vacuum states of byuons and the distance in the $R_{1}$ space. This distance is taken positive values only.

The proposed hypothesis requires to develop a mathematical model based on a new algebra of probabilistic events since the elementary events (a discrete decrease $(\overline{\mathrm{A}})$ or increase $(D)$ in the length of byuon) are assumed to be probabilistic in character. Hence for the byuons of the minimum length we may say about the existence, with certain probability, of the events $\overline{\mathrm{A}} \cdot D$ $\neq 0$. Note that in [9] an algebra of events is given, being a development of the Boolean algebra with the proviso that $\ddot{\mathrm{A}} \cdot D=1$. For the deterministic approach used in [28], the event $\bar{A} \cdot D \neq 0$ is illogical by von Neumann, but in the probabilistic space of events the existence of $\overline{\mathrm{A}} \cdot D \neq$ 0 is possible.

In this paper only the physical statement of the problem will be considered, and results of evaluations made in support of the hypothesis advanced, will be given.

The space $R_{1}$ is formed from the set of byuons in such a manner that at its $i$-th point there exist all the byuons with the lengths smaller than $X(i)$ or equal to $X(i)$ for $X(i)$ $>0$, and those with the absolute values smaller than $X(k-i)$ for $X(k-i)<0$, where $k$ is some period in $i$.

The assumption that two neighboring byuons (the $i$-th and $(i+1)$-th; $(i+1)$-th and $(i+2)$-th etc.) being in vacuum states $I^{+}$will interact, is unreasonable since in this case the definition of byuons for this VS would be violated at the point of interaction. Such interaction is possible only between the $i$-th and $(i-k)$-th byuons in the state $I I^{+}$if they form a "loop" in the space $R_{1}$ (by the "loop", the periodicity of the process in $i$ is implied), i.e. the two byuons $\mathrm{II}_{i}^{+}$ and $I I_{i-k}^{+}$will be observed simultaneously at one point of the space $R_{1}$. The least possible value of $k$ is $k=3$. In Figure 2 shown is the interaction of byuons in the vacuum states $\mathrm{II}_{1}^{+}$and $\mathrm{II}_{4}^{+}$(the smallest loop). The byuons in the state $I \Gamma$ interact likely.

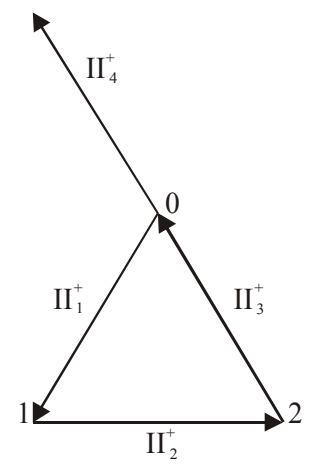

Figure 2. Interaction of byuons in vacuum states $\mathrm{II}_{1}{ }^{+}$and $\mathrm{II}_{4}^{+}$(the smallest loop).
The byuon states $I^{+}$and $I$ can occur only if the byuons have already been in VS $I I^{+}$and $I I$, respectively. At maximum positive potential energy of byuon interaction there exists a single variant of "occupancy" (Figure 3).

The probability of the minimum four-contact interaction of the neighboring in $i$ byuons $I^{+} I I^{+} \times I I I$ (" $\times$ " symbolizes interaction) with randomly appearing states $I^{+}$and $I$, is equal to $1 / 16$ [20-22]. That is quite understandable when analyzing possible four-contact interactions (see Figure 4)). All other possible variants of the four-contact interaction are unobservable either because one cannot introduce them without violating the definition of byuons or in view of imaginary energy of such interaction.

Note once more that there exist only two directions in the one-dimensional world, the first of which corresponds to increasing index $i$ for byuons with $X>0$ (vacuum $I \Gamma^{+}$), and the second corresponds to decrease in $i$ for

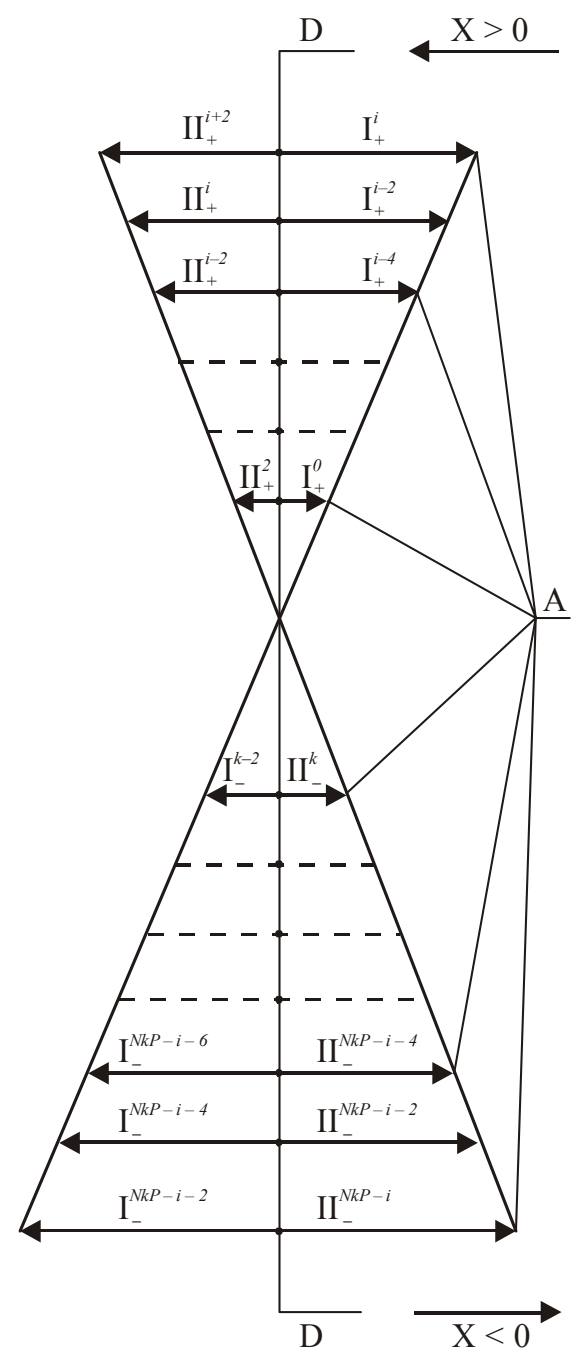

Figure 3. Completion of vacuum states $I^{+}$and $I \Gamma$ by vacuum states $I^{+}$and $I$, respectively, at the maximum potential energy of interaction. 

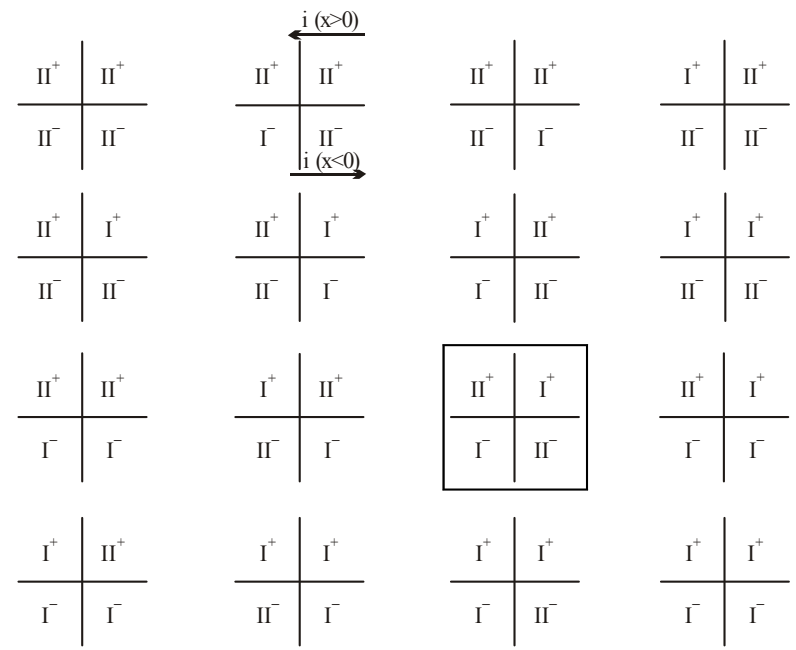

Figure 4. The possible variants of four-contact interaction of byuons. Square means that this interaction can realize in nature.

such byuons (vacuum $I^{+}$). These directions are coincident with those for byuons with $X<0$ : $I \Gamma$ with $I^{+}$, and $I$ with $I I^{+}$. It is clear from above definitions that the byuon with the maximum length $X(i)$ in VS $I I^{+}$determines the positive direction, and directions of other byuons are correspondent with it.

The four-contact interaction of byuons is realized within a time $\tau=\widetilde{\tau}_{0}$ only at points $D$ of the $R_{1}$-space

(Figure 3), i.e. at the points where introducing an interaction with $P E>0$ is possible. In Figure 3 the arrows corresponding to byuons show directions of a decrease or an increase in their lengths relative to the origin of the coordinates introduced, for example, where $i \rightarrow 0$ (in its direction the absolute value of the byuon length decreases (states $I^{+}$and $I$ ), and it increases in the opposite directions (states $I I^{+}$and $I I^{-}$)). At the points $A$ in Figure 3 , the coordinate denoting place (time) of byuon interaction cannot be fixed because of violating, in such a case, the definitions of the byuon states (in one quantum of the $R_{1}$-space within a time $\tau_{0}$, the byuons with $I I^{+} I^{+} I I I$ should not be present). It is assumed that before the origin of VS $I^{+}$with the minimum length $(i=1)$, the byuon vacuum states $I I^{\top}$ and $I^{\top}$ with any possible lengths are already in existence.

The propagation of byuons in VSs $I I^{+} I^{+}$and $I I I$, the interaction between which occurs with imaginary energy (see below ), presents two wave-like processes (see below) directed towards each other at $X(i)>0$ and $X(i)<0$, respectively. These processes are unobservable. A really observable signal can be transmitted by means of such processes only in the four-contact byuon interaction $I I^{+} I^{+} \times$ III.

Let us obtain an equation characterizing the propagation of the four-contact interaction of byuons in $R_{1}$. Introduce functions of index $i$, characterizing the origin of such or another VS by byuons: $\Psi_{I^{+}}^{i+2}, \Psi_{I^{-}}^{k-i}$, determining the processes of byuon length magnitude origin and increase at positive and negative $X(i)$, respectively; $\Psi_{I^{+}}^{i}, \Psi_{I^{-}}^{k-i-2}$, determining the processes of byuon length magnitude cancellation and decrease at positive and negative $X(i)$, respectively.

The physical sense of the introduced functions consists in that their product determines the probability of two-contact interaction of byuons (for example, $\Psi_{I^{+}}^{i+2}$. $\Psi^{i+2-k}$ determines the probability of interaction of byuons $[A \cdot X]_{I I^{+}}^{i+2}$ и $[A \cdot X]_{I I^{+}}^{i+2-k}$ ), the product of four functions determines the probability of four-contact interaction, the product of eight functions gives the probability of eight-contact interaction. These products should be positive, and in this case only they can describe an observed event.

The probability of a single event is no greater than 1 .

Depending on which range is $i$ in $(0 \leq i<k, k<i<$ $N k, N k<i<N k P$ where $k, N, P$ are the assumed periods in $i$ ) various types of contact interactions between byuons may be introduced. Hence the normalization of the introduced functions should be dependent on $i$.

Let us normalize the introduced functions for the case $0 \leq i<k$ in the following manner

$$
\begin{gathered}
\sum_{\xi=0}^{(N k P-k) / 2} \sum_{j=0}^{j=i} \Psi_{I I^{+}}^{j+2} \cdot \Psi_{I^{+}}^{j} \cdot \Psi_{I I^{-}}^{N k P-j-2-2 \xi} \cdot \Psi_{I^{-}}^{N k P-j-2-2 \xi}=\frac{N P}{2} \\
\sum_{\xi=1}^{N P-1} \sum_{j=0}^{j=i} \Psi_{I I^{-}}^{N k P-j} \cdot \Psi_{I I^{-}}^{N k P-j-2-\xi k}=P, \\
\sum_{\xi=0}^{(N k P-k) / 2} \sum_{j=0}^{j=i} \Psi_{I^{+}}^{j+2} \cdot \Psi_{I^{-}}^{N k P-j-2-2 \xi}=\frac{N P}{2}, \\
\sum_{\xi=0}^{N k P-k) / 2} \sum_{j=0}^{j=i} \Psi_{I^{+}}^{j} \cdot \Psi_{I I^{-}}^{N k P-j-2-2 \xi}=\frac{N P}{2} .
\end{gathered}
$$

When normalizing, it is taken into account that within a period in $i=k$, one four-contact interaction occurs with probability 1 .

Let us obtain an equation in terms of $\Psi$-functions, describing the propagation of a four-contact interaction of byuons. For that we may write the following relationships as to the origin of VSs $I I^{+}\left(f_{I I^{+}}\right), I^{+}\left(f_{I^{+}}\right), I I^{-}\left(f_{I I^{-}}\right)$, $I^{-}\left(f_{I^{-}}\right)$depending on certain VSs of the byuons neighbouring in the index $i$ :

$$
\begin{aligned}
& \Psi_{I^{+}}^{i+2}=f_{I I^{+}}\left[\Psi_{I+}^{i+1}, \Psi_{I^{+}}^{i+2}, \Psi_{I I^{-}}^{N k P-i}, \Psi_{I^{-}}^{N k P-i-2}\right], \\
& \Psi_{I^{+}}^{i}=f_{I^{+}}\left[\Psi_{I^{+}}^{i+1}, \Psi_{I I^{+}}^{i}, \Psi_{I I^{-}}^{N k P-i}, \Psi_{I^{-}}^{N k P-i-2}\right], \\
& \Psi_{I I^{-}}^{N k P-i}=f_{I I^{-}}\left[\Psi_{I I^{-}}^{N k P-i-1}, \Psi_{I^{-}}^{N k P-i-1}, \Psi_{I I^{+}}^{i+2}, \Psi_{I^{+}}^{i}\right], \\
& \Psi_{I^{-}}^{N k P-i-2}=f_{I^{-}}\left[\Psi_{I^{-}}^{N k P-i-1}, \Psi_{I I^{-}}^{N k P-i-2}, \Psi_{I I^{+}}^{i+2}, \Psi_{I^{+}}^{i}\right] .
\end{aligned}
$$


Assuming only linear dependences in Expressions (9) as well as equiprobability of VSs of byuons neighbouring in $i$, we obtain the following equations for $\Psi$-functions of four-contact interactions of byuons:

$$
\begin{aligned}
& \Psi_{I I^{+}}^{i+2}=\Psi_{I I^{+}}^{i+1}-\Psi_{I^{+}}^{i+2}-\Psi_{I I^{-}}^{N k P-i}+\Psi_{I^{-}}^{N k P-i-2}, \\
& \Psi_{I^{+}}^{i}=\Psi_{I^{+}}^{i+1}-\Psi_{I I^{+}}^{i}+\Psi_{I I^{-}}^{N k P-i}-\Psi_{I^{-}}^{N k P-i-2}, \\
& \Psi_{I I^{-}}^{N k P-i}=\Psi_{I I^{-}}^{N k P-i-1}-\Psi_{I^{-}}^{N k P-i-1}-\Psi_{I I^{+}}^{i+2}+\Psi_{I^{+}}^{i}, \\
& \Psi_{I^{-}}^{N k P-i-2}=\Psi_{I^{-}}^{N k P-i-1}-\Psi_{I I^{-}}^{N k P-i-2}+\Psi_{I I^{+}}^{i+2}-\Psi_{I^{+}}^{i} .
\end{aligned}
$$

From the first and second pairs of Equations (10) we obtain, respectively, the following equations:

$$
\begin{aligned}
& \Delta\left[\Psi_{I I^{+}}^{i+1}+\Psi_{I^{+}}^{i+1}\right]+\Psi_{I I^{+}}^{i+1}+\Psi_{I^{+}}^{i+1}=0, \\
& \Delta E(i)=\Psi_{I I^{-}}^{N k P-i-k} \Psi_{I I^{-}}^{N k P-i} E_{I I^{-} I I^{-}}^{N k P-i-k, N k P-i} \cos _{I I^{-} I I^{-}}+\Psi_{I I^{-}}^{N k P-i-2 k} \Psi_{I^{-}}^{N k P-i} E_{I I^{-} I I^{-}}^{N k P-i-2 k, N k P-i} \cos _{I I^{-} I I^{-}}+ \\
& +\Psi_{I I^{-}}^{N k P-i-3 k} \Psi_{I I^{-}}^{N k P-i} E_{I I^{-} I I^{-}}^{N k P-i k, N k P-i} \cos _{I I^{-} I^{-}}+\ldots+\Psi_{I^{+}}^{i+2} \Psi_{I^{-}}^{N k P-i-2} E_{I I^{+} I^{-}}^{i+2, N k P-i-2} \cos { }_{I I^{+} I^{-}}+ \\
& +\Psi_{I I^{+}}^{i+2} \Psi_{I^{-}}^{N k P-i-2-2} E_{I^{+} I^{-}}^{i+2, N-i-2-2} \cos { }_{I I^{+} I^{-}}+\Psi_{I^{+}}^{i+2} \Psi_{I^{-}}^{N k P-i-2-2 \times 2} E_{I^{+} I^{-}}^{i+2, N k-i-2-2 \times 2} \cos _{I^{+} I^{-}}+ \\
& +\Psi_{I I^{+}}^{i+2} \Psi_{I^{-}}^{N k P-i-2-2 \times 3} E_{I I^{+} I^{-}}^{i+2, N k P-i-2-2 \times 3} \cos _{I I^{+} I^{-}}+\ldots+\Psi_{I I^{-}}^{N k P-i} \Psi_{I^{+}}^{i} E_{I I^{-} I^{+}}^{N k P-i, i} \cos _{I I^{-} I^{+}}+\Psi_{I I^{-}}^{N k P-i-2} \Psi_{I^{+}}^{i} E_{I I^{-} I^{+}}^{N k-i-2, i} \cos _{I I^{-} I^{+}}+ \\
& +\Psi_{I I^{-}}^{N k P-i-2 \times 2} \Psi_{I^{+}}^{i} E_{I I^{-} I^{+}}^{N k P-i-2 \times 2, i} \cos _{I I^{-} I^{+}}+\Psi_{I I^{-}}^{N k P-i-2-3} \Psi_{I^{+}}^{i} E_{I I^{-} I^{+}}^{N k P-i-2 \times 3, i} \cos I I_{I^{-} I^{+}}+\ldots+ \\
& +\Psi_{I^{+}}^{i} \Psi_{I I^{+}}^{i+2} \Psi_{I^{-}}^{N k P-i-2} \Psi_{I I^{-}}^{N k P-i} \sqrt{E_{I^{+} I I^{+}}^{i, i+2} \cos _{I^{+} I I^{+}} E_{I^{-} I I^{-}}^{N k P-i-2, N k P-i} \cos _{I^{-} I I^{-}}}+ \\
& +\Psi_{I^{+}}^{i} \Psi_{I I^{+}}^{i+2} \Psi_{I^{-}}^{N k P-i-2-2} \Psi_{I I^{-}}^{N k P-i-2} \sqrt{E_{I^{+} I I^{+}}^{i, i+2} \cos _{I^{+} I I^{+}} E_{I^{-} I I^{-}}^{N k P-i-2-2, N k P-i-2} \cos _{I^{-}} I I^{-}}+ \\
& +\Psi_{I^{+}}^{i} \Psi_{I I^{+}}^{i+2} \Psi_{I^{-}}^{N k P-i-2-2 \times 2} \Psi_{I I^{-}}^{N k P-i-2 \times 2} \sqrt{E_{I^{+} I I^{+}}^{i, i+2} \cos { }_{I^{+} I I^{+}} E_{I^{-} I I^{-}}^{N k P-i-2-2 \times 2, N k P-i-2 \times 2} \cos _{I^{-} I^{-}}}+ \\
& +\Psi_{I^{+}}^{i} \Psi_{I I^{+}}^{i+2} \Psi_{I^{-}}^{N k P-i-2-2 \times 3} \Psi_{I I^{-}}^{N k P-i-2 \times 3} \sqrt{E_{I^{+} I I^{+}}^{i, i+2} \cos _{I^{+} I I^{+}} E_{I^{-} I I^{-}}^{N k P-i-2-2 \times 3, N k P-i-2 \times 3} \cos _{I^{-} I I^{-}}}+\ldots+
\end{aligned}
$$

where $E_{I^{-} I I^{-}}^{N k P-i-k, N k P-i}, \ldots, E_{I^{+} I I^{+}}^{i, i+2}$, etc. are maximum values of potential energy of interactions of byuons with the lengths $X(N k P-i-k)<0$ and $X(N k P-i)<0$ in VS $I \Gamma$, as well as byuons with the lengths $X(i)$ and $X(i+2)$ in VSs $I^{+}$ and $I I^{+}$, respectively; $\cos _{I^{+} I^{+}}, \cos _{H^{-I I}}$ etc. are functions minimizing the potential energy of interactions of byuons entering into the expressions for $E_{I^{+} I I^{+}}^{i, i+2}, E_{I^{-} I I^{-}}^{N k P-i, N k P-i}$, etc.; these functions are "responsible" for the appearance of a minimum plane object and the introduction of the concept of spin (see below). Functions $\cos _{I^{+} I^{+}}$are not the usual Cos-functions, because we work up to now in one-dimensional space of $\mathrm{i}$-indices. These Cos $\backslash$ s can have values from 0 to 1 . Upper and lower indices in (13) correspond to interactions of byuon VSs,

The difference in the average values of byuon lengths calculated basing upon the definition of byuons with the use of the rule of circular arrow (see Figure 5), is taken as a distance between the interacting byuons to find $\Delta E(i)$ $>0$. The meaning of this rule is that the said distance is calculated as the difference in the length values of the subsequent and preceding byuons in the direction pointed by an arrow.

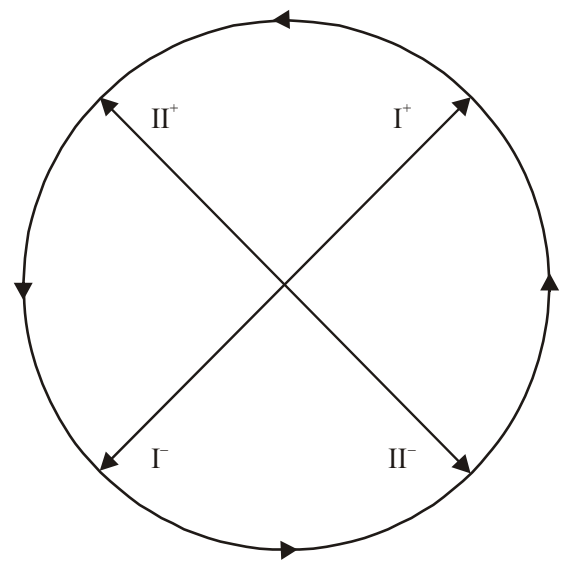

Figure 5. The rule of circular arrow for determining the distance between byuons. 
The lengths of byuons are:

$X_{I_{c p}^{+}}^{i+1}=\frac{2 i+1}{2} \cdot \frac{\text { const }_{1}}{k \cdot A_{G}} ; \quad X_{I_{c p}^{-}}^{k-i}=-\frac{2(k-i)-1}{2} \cdot \frac{\text { const }_{1}}{k \cdot A_{G}} ;$

$X_{I_{c p}^{+}}^{i}=\frac{2 i+1}{2} \cdot \frac{\text { const }_{1}}{k \cdot A_{G}} ; \quad X_{I_{c p}^{-}}^{k-i-1}=-\frac{2(k-i)-1}{2} \cdot \frac{\text { const }_{1}}{k \cdot A_{G}}$

etc.

The distance between byuons does not depend on $i$ and may take by magnitude only two values:

$$
\begin{array}{ll}
X_{I I^{-} I^{+}}^{k-i, i}=\frac{\text { const }_{1}}{A_{\mathrm{G}}} ; & X_{I I^{+} I^{-}}^{i+1, k-i-1}=-\frac{\text { const }_{1}}{A_{\mathrm{G}}} ; \\
X_{I^{+} I I^{+}}^{i, i+2}=\frac{\text { const }_{1}}{k \cdot A_{\mathrm{G}}} ; & X_{I^{-} I I^{-}}^{k-i-2, k-i}=-\frac{\text { const }_{1}}{k \cdot A_{\mathrm{G}}} ;
\end{array}
$$

or multiples of them, for example,

$$
X_{I I^{-} I^{+}}^{N k-i, i}=\frac{\text { const }_{1} N}{A_{\mathrm{G}}} .
$$

The expressions determining the maximum energy of byuon interactions are written as

$$
\begin{aligned}
& E_{I I^{-} I^{+}}^{k-i, i}=\frac{[A x]_{I I^{-}}^{k-i}[A x]_{I^{+}}^{i}}{X_{I I^{-} I^{+}}^{k-i, i}}=\frac{\text { const }_{1}}{4 k^{2}} A_{\mathrm{G}}[2(k-i)-1](2 i+1) ; \\
& E_{I I^{+} I^{-}}^{i+2, k-i-2}=\frac{\text { const }_{1}}{4 k^{2}} A_{\mathrm{G}}[2(k-i)-1](2 i+1) ; \\
& E_{I^{+} I I^{+}}^{i, i+2}=-\sqrt{-1} \frac{\text { const }_{1}}{4 k^{2}} A_{\mathrm{G}}(2 i+3)(2 i+1) ; \\
& E_{I^{-} I I^{-}}^{k-i-2, k-i}=\sqrt{-1} \frac{\text { const }_{1}}{4 k^{2}} A_{\mathrm{G}}[2(k-i)-1][2(k-i)-3] ; \\
& E_{I I^{-} I I^{-}}^{N k P-i-k, N k P-i}=\frac{\text { const }_{1}}{4 k^{2}} A_{\mathrm{G}}[2(N k P-i-k)-1][2(N k P-i)-1] .
\end{aligned}
$$

The minimization of $\triangle E(i)$ is achieved in the functional space of the following variables:

$$
\Psi_{I^{+}}^{0}, \Psi_{I I^{+}}^{2}, \Psi_{I I^{-}}^{N k P}, \Psi_{I^{-}}^{N k P-2}, \cos _{I^{+} I I^{+}}^{i, i+2}, \cos _{I I^{-} I I^{-}}^{N k P-k, N k P-i}, k, N P
$$

It is assumed therewith that the conditions of symmetry during the "closure of the loop" in $i$ are fulfilled as well as symmetry of the world and antiworld, which conditions can be represented as:

$$
\begin{gathered}
\cos _{I^{+} I I^{+}}^{i, i+2}=\cos _{I^{-} I I^{-}}^{N k P-i-2, N k P-i}=\cos _{I^{-} I I^{-}}^{N k P-i-2-2, N k P-i-2} \\
=\cos _{I^{-} I I^{-}}^{N k P-i-2-2 \times 2, N k P-i-2 \times 2}=\ldots ; \\
\cos _{I I^{-} I I^{-}}^{N k P-i-k, N k P-i}=\cos _{I I^{-} I I^{-}}^{N k P-i, 2 k, N k P-i}=\ldots ;
\end{gathered}
$$

The functions $\cos _{I I^{+} I^{-}}, \cos _{I I-I^{+}}$are considered as equal to 1 .

Initial conditions for $\Psi$ - function are preset to be

$$
\begin{aligned}
& \Psi_{I I^{+}}^{0} \approx 0, \Psi_{I^{+}}^{0} \approx 1, \Psi_{I I^{-}}^{N k P} \approx 1, \Psi_{I^{-}}^{N k P} \approx 0, \Psi_{I^{+}}^{1} \\
& \approx \Psi_{I^{-}}^{N k P-2}, \Psi_{I I^{-}}^{N k P-1}=\Psi_{I I^{+}}^{2}, \Psi_{I^{-}}^{i+2}+\Psi_{I^{-}}^{i+1}=0
\end{aligned}
$$

Using the solutions of the Equations (11) and (12): $\Psi_{I I^{+}}^{i+1}+\Psi_{I^{+}}^{i+1}=A \cos \left(\frac{2 \pi i}{k}\right)+B \sin \left(\frac{2 \pi i}{k}\right)$, we contract the space of variables down to four: $\cos _{I^{+} I I^{+}}^{i, i+2}$, $\cos _{I I^{-} I I^{-}}^{N k P-i-2, N k P-i}, k, N P$.

Now, taking into account the normalizing Expressions (5-8), seek for $\min \Delta E(i)$ by the steepest descent method. When retaining only 14 terms of the series in (16) and (5), $\min \Delta E(i)$ will correspond to the following values:

$$
\begin{aligned}
& \Psi_{I^{+}}^{0}=0.999(6), \quad \Psi_{I I^{+}}^{2}=1.00136 \times 10^{-4}, \quad \Psi_{I I^{-}}^{N k P}=0.999(8), \\
& \Psi_{I^{-}}^{N k P-2}=1.100043 \times 10^{-4}, \quad \cos _{I^{+} I I^{+}}^{i, i+2}=1.01887 \times 10^{-5}, \\
& \cos _{I I^{-} I I^{-}}^{N k P-i-k, N k-i}=1.20013 \times 10^{-5}, \quad k= \\
& 6.2 \times 10^{15}, \quad N k P=3 \times 10^{60}
\end{aligned}
$$

With increasing $n$, as is seen from the solutions given and Figure 6, $k$ (the first period in $i$ ) approaches its value obtained in $[25,26]$ on the base of physical considerations as an integer part of the ratio $x_{0} / \tilde{x}_{0}=.3 .2 \times 10^{15}$ Thus, we can now obtain, with the aid of the calculated $k$, one of the fundamental dimensions in physics of elementary particles, $x_{0} \approx 10^{-17} \mathrm{~cm}$, with the only quantum of space $\tilde{x}_{0}$ given. This mathematical result raises prospects that the advanced hypothesis is true. It reflects the nature of physical space and vacuum.

The minimum $\Delta_{1} E(i)$ was sought for a case when $N k<$ $i \leq N k P$. In this case the normalizing expressions for the arising interactions of byuons have the form:

$$
\sum_{j=N k}^{j=i} \sum_{\xi=0}^{\xi=\frac{N k P-i-2}{2}} \Psi_{I^{+}}^{j} \Psi_{I I^{+}}^{j+2} \Psi_{I^{-}}^{N k P-j-2-2 \xi} \Psi_{I I^{-}}^{N k P-j-2 \xi}=\frac{i N P}{2 k}
$$

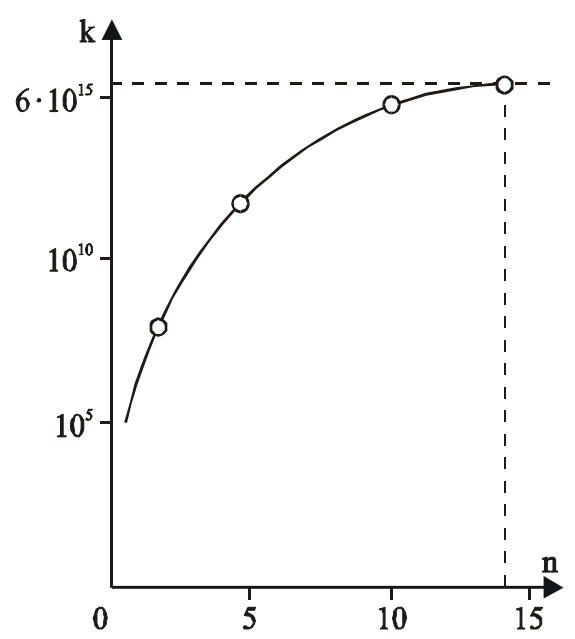

Figure 6. $k$ as a function of the number $n$ of the terms of the series. 


$$
\begin{array}{r}
\xi=\frac{N k P-i-2}{\sum_{\xi=1}^{2}} \Psi_{I I^{-}}^{N k P-i} \Psi_{I I^{-}}^{N k P-i-\xi k}=\frac{N k P-i}{N k} \\
\sum_{\eta=1}^{i / k} \Psi_{I I^{+}}^{i+2} \Psi_{I I^{+}}^{i+2-\eta k}=\frac{i}{N k}
\end{array}
$$

$$
\begin{gathered}
\xi=\frac{N k P-i-2}{\sum_{\xi=0}^{2}} \sum_{j=N k}^{j=i} \Psi_{I I^{+}}^{j+2} \Psi_{I^{-}}^{N k P-j-2-2 \xi}=\frac{i N P}{2 k} \\
\sum_{\xi=0} \sum_{j=N k}^{N=i} \Psi_{I I^{-}}^{N k P-j-2 \xi} \Psi_{I^{+}}^{j}=\frac{i N P}{2 k} .
\end{gathered}
$$

The expression for $\Delta_{1} E(i)$ becomes more complicated:
( $\Delta E(i)$ is taken from Equation (10), taking into account for multiplication over index $i$ as was shown above $(i+2, I+$ $2-k, i+2-2 k$ etc.).

The search for $\min \Delta E(i)$ with the use of the chain of Equations (7) in the space of similar variables with similar initial conditions (where $i=0$ corresponds now to $i=$ $N k$ ), leads to practically the same results:

$$
\begin{aligned}
& \Psi_{I^{+}}^{N k}=0.9998, \quad \Psi_{I I^{+}}^{N k+2}=1.006 \times 10^{-4}, \quad \Psi_{I I^{-}}^{N k P} \\
& =0.9999, \\
& \Psi_{I^{-}}^{N k P-2}=1.101 \times 10^{-4}, \quad \cos _{I^{+} I I^{+}}^{i, i+2}=\cos _{I I^{+} I I^{+}}^{i+2, i+2-k} \\
& =1.1019 \times 10^{-5}, \\
& \cos _{I I^{-} I I^{-}}^{N k P-i-k, N k p-i}=1.203 \times 10^{-5}, \quad k=4.475 \times 10^{15}, \\
& =3.169 \times 10^{60}
\end{aligned}
$$

It is interesting that at $i<k$ and even at $i \approx N k$ (Table 1), a significant part in the magnitude of $\Delta E(i)$ is potential energy of the byuon interaction in $\mathrm{VS} I I^{-} I I^{-}$, and at $i$ $\rightarrow N k P$ the four-contact interaction of byuons in VS $\mathrm{II}^{+} \mathrm{I}^{+} \mathrm{I}^{-} \mathrm{II}^{-}$becomes determining.

Let us consider a simplified case when only the period in $i$, equal to $k$, is present in the antiworld. Then we have from the necessary condition of minimum $\Delta E(i)$ with respect to the function $\Psi_{I I^{+}}^{i+2}$ (i.e. from the equation $\left.\frac{\partial \Delta E(i)}{\partial \Psi_{I I^{+}}^{i+2}}=0\right)$ :

Table 1. The values of potential energy $E$ in vacuum states $\left(\begin{array}{ll}\left(I^{+}\right. & \mathrm{II}^{+}\end{array}\right),\left(\mathrm{II}^{+} \mathrm{I}^{+} \mathrm{I}^{-} \mathrm{II}^{-}\right),\left(\mathrm{II}^{-} \mathrm{II}^{-}\right)$depending on the $\mathrm{i}$ index.

\begin{tabular}{ccc}
\hline $\mathrm{E}[\mathrm{erg}]$ & $\mathrm{Nk}$ & $\mathrm{NkP}$ \\
\hline$E_{I I^{+} I I^{+}}$ & $10^{13}$ & $10^{95}$ \\
$E_{I I^{+} I^{+} I^{-} I I^{-}}$ & $10^{70}$ & $10^{111}$ \\
$E_{I I^{-} I I^{-}}$ & $10^{95}$ & $10^{13}$ \\
\hline
\end{tabular}

$\cos _{I I^{+} I^{+}}=-\frac{1}{\Psi_{I^{+}}^{i} \cdot \Psi_{I I^{-}}^{k-i}} \cdot \frac{1}{k} \cdot \frac{\sqrt{[(k-i)-1](2 i+1)}}{(2 i+3)[(2 k-i)+3]}$.

If $i<<k, N=P=1$, and hence, according to Equation (5) $\sum_{j=0}^{j=i} \Psi_{I^{+}}^{j} \cdot \Psi_{I I^{-}}^{k-j} \rightarrow 1$, we obtain from Equation (20) $\cos _{I I^{+} I^{+}} \approx 1 / k$.

Thus, as show our numerical calculations and analytical estimations, the minimization of $\Delta E(i)$ leads to values of functions $\cos _{\mathrm{II}^{+} \mathrm{I}^{+}}$etc. if not zero but extremely small.

Note that the functions $\cos _{\mathrm{II}^{+} I^{+}}$for the case of $N P$ "loops" in VS $I I^{-}$(see (18)) and for the case when $N=P$ $=1$, differ by ten orders of magnitude. The physical meaning of these functions will be shown further.

Put a question, where disappears and into what kind of energy the potential energy of byuon interaction transforms? An answer seems to be simple, of course, - into the kinetic energy of rotation (since $\cos _{I I^{+} I^{+}}<<1$, see (18, 20)). But the rotation of what and around what? And why do we assume the law of conservation of energy to be fulfilled here? After all, it makes no sense to say about uniformity in time for this statement in which the time is discrete! Let us answer these questions.

As it was shown above, the optimum values of the functions $\cos _{I I^{+} I^{+}}^{i+2,}, \cos _{I I^{-} I^{-}}^{N k P-i, N k P-i-2}$, etc., are much less than 1 but non-zero. The smallest values of $\cos _{I^{+} I^{+}} \approx 1 / k$ correspond to residual (finite) potential energies $E_{k}$, from which, as we will further assume, the smallest part (associated with the formation of the own space of elementary particles) of the potential self-energy of elementary particles corresponding to the known Einstein's relationship $E_{k}=m c^{2}$, is added together. Determine the minimum value of $E_{k}$. It is seen from Equation (13) that for the 
simplest objects with $N$ and $P$ approximately equal to 1 , the minimum $E_{k}$ is equal to the potential energy of the four-contact interaction of byuons with the minimum values of $\cos _{I I^{+} I^{+}}, \cos {I I-I^{-} \text {. }}$.

In view of the normalization (5) we have for this case

$$
E_{k_{\min }}^{(0)}=\sqrt{E_{I^{+} I I^{+}}^{0,2} \cos _{I^{+} I I^{+}} E_{I^{-} I I^{-}}^{k-2, k} \cos _{I^{-} I I^{-}}} .
$$

From that, with the condition (16), and using Equation (14) and the equality $\cos _{I I^{+} I^{+}} \approx 1 / k$, we obtain $E_{k_{\min }}^{(0)} \approx$ $33 \mathrm{eV}$.

Consider the process of energy transformation for the four-contact byuon interaction occurring within a time quantum $\tau_{0}$ (Hence, the transformation must be discrete).

Any value of $i$ can be redenoted with an another index, for example, with $j, \gamma$, $\xi$, etc., which may be set equal to zero. At each point then, where $j=\gamma=\xi=\ldots=0$, there will be always present an own system of account of the indices $j, \gamma, \xi$, etc., as well as the minimum energy of four-contact byuon interaction $E_{k_{\min }}^{(0)} \approx 33 \mathrm{eV}$. Note that this minimum energy is limited in index $i$ by values $i=0$ and $i=2$ (point $A$ Figure 3).

Thus, we have in $R_{1}$ two sets of points $A(\{A\})$ and $D(\{D\})$, between which the dynamic process of renumeration goes in connection with the properties of vacuum states of the discrete objects, the byuons $I I^{+}, I^{+}, I, I \Gamma$ :

$$
\begin{gathered}
\text { the time } \tau_{i+1}\left\{\begin{array}{l}
\{D\}=i+1, i-1, \ldots \\
\{A\}=i+2, i, \ldots
\end{array}\right. \\
\text { the time } \tau_{i+1}+\tau_{0}\left\{\begin{array}{l}
\{D\}=i+2, i, \ldots \\
\{A\}=i+3, i+1, \ldots
\end{array}\right.
\end{gathered}
$$

The set $R_{1}$ may be represented, at some $i$-th point of time as the join of $\{A\}$ and $\{D\}$, i.e. $R_{1}=\{A\} U\{D\}$ (Figure 7).

Hence the space $R_{1}$ segregates into the subspaces $R_{D}$ of $D$-points and $R_{A}$ of $A$-points. Thus, we may say about the motion of $D$-points relative to $A$-points. A new, second coordinate appears, symbolize it by $Y_{A D}$ (Figure 7).

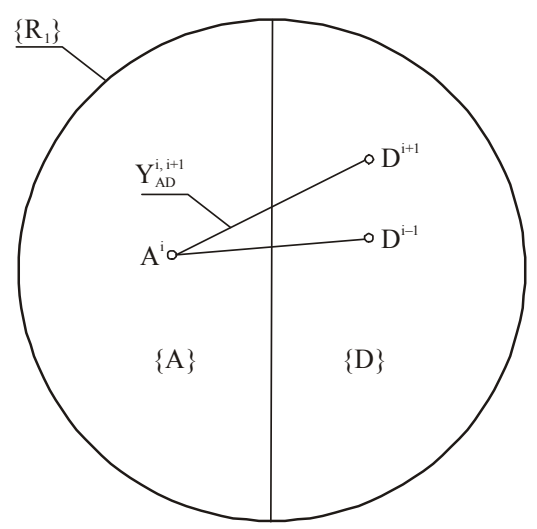

Figure 7. Representation of the set $\left\{R_{1}\right\}$ as a union of sets of the points $\{A\}$ and $\{D\}$.
The minimum value of $Y_{A D}: Y_{A D_{\min }}=C_{0} \tau_{0} \mid(i+1)_{A}$ $-i_{D} \mid=\widetilde{X}_{0}$. The minimum object appears. Assume that its appearance (new coordinate) corresponds to the minimum action $h\left(\right.$ see $[1-3], \mathrm{h}=\left(\left(\left[\mathrm{A}_{\mathrm{\Gamma}} \mathrm{x}_{\mathrm{o}}\right]_{\mathrm{II}}^{+}\left[\mathrm{A}_{\Gamma} \mathrm{x}_{\mathrm{O}}\right]_{\mathrm{I}}{ }^{-}\right) / \mathrm{c}_{\mathrm{o}}\right)$ $\mathrm{x}_{\mathrm{o}} / \mathrm{ct}^{*}$ and elementary electric charge $\mathrm{e}_{\mathrm{o}}{ }^{2}=(1 /(4 \sqrt{ } 3))$ $\left.\mathrm{A}_{\mathrm{g}}{ }^{2} \mathrm{x}_{\mathrm{o}}{ }^{2}\left(\mathrm{x}_{\mathrm{o}} / \mathrm{ct}^{*}\right)^{3 / 2}\right)$. We may then introduce the concept of momentum for objects with the residual PE of a byuon interaction by writing the relationship $P_{D}^{i+1} \times Y_{A D}^{i, i+1}=h$ where $P_{D}^{i+1}$ is the momentum of the point $D$ numbered $i+1$ relative to the point $A$ with the number $i$. Similar relationship can be written in any point from $\{A\}$. The direction of the momentum vector $\vec{P}_{D}^{i+1}$ corresponds to that towards the point $D^{i+3}$ of the subspace $R_{D}$. The direction of the coordinate $Y_{A D}^{i, i+1}$ corresponds to the vector directed from the point $A^{i}$ to the point $D^{i+1}$.

The appearance of the minimum plane object and realization of the minimum action are connected with the origin of the quantum spin number $\bar{S}=\left[\bar{P}_{D}^{i+1} \times \bar{Y}_{A D}^{i, i+1}\right]$, expressed numerically in minimum actions $h$.

The function $\cos _{I^{+} I^{+}}$etc. minimizing $\Delta E(i)$, will be further considered by us as cosines of the angles between the vector $\bar{P}_{D}^{i+1}$ and $\bar{Y}_{A D}^{i, i+1}$ i.e. before the byuon interaction, the space $R_{1}$ represents, at some time point $\tau$, a certain discrete straight line of points $\{D\}$ and $\{A\}$, and at the time point $\tau+\tau_{0}$ forms a line, broken at points $\{D\}$. That is the minimum interaction of byuons has occurred.

If an "observer" was able to perceive objects with $E>0$ only every $\tau=k \tau_{0}$, he would simultaneously (within a time quantum $\tau_{0}$ ) fix all the planes arrived to the point of "observation", and "see" already the three-dimensional world formed from the plane world in the result of its dynamics within the time $\tau=\tau_{0}$. Why the three-dimensional and not $\mathrm{N}$-dimensional one? Because the set of two-contact interactions of byuons is divided, depending on reference point (Figure 2, $i=0,1,2$ ), into three subsets $M_{0}, M_{1}, M_{2}$ (the lower index denotes reference point), corresponded by three one-dimensional subspaces $R_{1,0}$, $R_{1,1}, R_{1,2}$ while introducing metric properties. Explain the above said.

As was indicated above, any value of index $i$ can be always redenoted by $j$ and then $j=0,1,2$ corresponds to reference points. Redenoting $i+1$ by $\xi, i+2$ by $\gamma$ etc. leads, depending on reference points, to formation of three families of subspaces embedded in each other:

$$
\begin{aligned}
& \text { reference point " } 0 \text { ” } \Rightarrow R_{1,0}^{i} \supset R_{1,0}^{j} \supset R_{1,0}^{\xi} \supset R_{1,0}^{\gamma} \text { etc.; } \\
& \text { reference point " } 1 \text { " } \Rightarrow R_{1,1}^{i} \supset R_{1,1}^{j} \supset R_{1,1}^{\xi} \supset R_{1,1}^{\gamma} \quad \text { etc.; } \\
& \text { reference point " } 2 \text { " } \Rightarrow R_{1,2}^{i} \supset R_{1,2}^{j} \supset R_{1,2}^{\xi} \supset R_{1,2}^{\gamma} \quad \text { etc., if }
\end{aligned}
$$
$i>j>\xi>\gamma$ etc.

Thus, in connection with the existence of three independent reference points for the new pair interactions, 
the three independent coordinates should be given to fix a pair interaction with respect of the three reference points, i.e. $R_{3}$ can be represented as $R_{3}=R_{1,0} \times R_{1,1} \times R_{1,2}$. Note that $R_{1,0}, R_{1,1}, R_{1,2}$ consist of the sets of points $\{A\}$ and $\{D\}$, i.e. at each subsequent point in time, renumeration of points $A$ and $D$ and "spinning" of objects with $E$ $>0$ in the subspaces $R_{1,0}, R_{1,1}, R_{1,2}$, occur. In this manner the concept of spin is introduced for objects with $E>0$ in $R_{3}$. For objects of a big size (as a result of described minimization of $\mathrm{PE}$ ), the rotation will be always take place since the byuons are not closed in a volume of $R_{3}$ due to $\cos _{I I^{+} I I^{+}} \neq 0$. That is why, planets, stars and so on rotate, with the main part of potential energy of byuons being transformed into energy of rotation.

Advance (without proving) the following theorem:

If a system is closed, the amount of information in it is constant.

That is, transformation of one information image into another is possible in the system, but the total amount of information does remain invariable.

Note that by information we mean here not informativity as in theory of information developed by Hartly and Shannon [29] on the basis of entropic approach, but the numbers of information bits (the values " 0 " and " 1 ") in one or another information subsystems of the system of considered objects (the combinatoric approach [30]). By " 1 " we imply here accomplishing the minimal act (minimum action $h / 2$ ) in the system with formation of an object with $E>0$ from byuons, and by " 0 " disappearing of the object with $E>0$ is meant.

On the base of the theorem, write the following equality for informational units (bits) in the subspaces $R_{1}$ and $R_{3}$ :

$$
2^{\frac{\tau_{0} E_{1} S_{1} k \cdot 2}{h}}=2^{S_{3} N}
$$

where $N$ is the number of information images in $R_{3}$ $\left(N=c t^{*} / x_{0}\right.$ - the second period in $\left.i\right) ; S_{3}$ the complex of the information image in $R_{3}$ (number of "loops" of length $\mathrm{N}$; $\left.S_{3}=1,2, \ldots\right) ; E_{1}$ potential energy of minimum four-contact interaction of byuons in $R_{1}$ if $\cos _{I I^{+} I^{+}}=\cos _{I I-I^{-}}=1$; $\frac{\tau_{0} E_{1} S_{1} \cdot 2}{h}$ is a transformation factor of recounting the number of information images $(k)$ in $R_{1}$ into that in $R_{3}$ for $i \geq k N ; S_{1}$ is complex of the information image in $R_{1}$ (number of "loops" of length $k, S_{1}=1,2, \ldots$ ).

By an information image in $R_{3}$, one means one or another quantum number of an elementary particle.

With Equation (22), the expressions for lepton masses obtained in [20-22] (see Appendix 1) become more understandable (for example, $m_{e} c^{2}=E_{k_{\min }}^{0} \cdot N=33 \mathrm{eV} \cdot \mathrm{ct}^{*} / x_{0}$ $\left.=2 m_{v_{e}} c^{2} \cdot c t^{*} / x_{0}\right)$.

Taking $k$ from the solution of the problem of searching the minimum $\Delta E$ and substituting $S_{3}=1, S_{1}=1$ into
Equation (22), we find $N=1.544 \times 10^{4}=c t^{*} / x_{0}$, and knowing $N$ and $N P$ from this solution, determine $P \approx$ $10^{42}$. The value of $\tilde{x}_{0} \mathrm{kN} \approx 10^{-13} \mathrm{~cm}$.

Thus, we find all the periods of byuon motion in $i$, corresponding to the following scales of our World: $10^{-17}$ $\mathrm{cm}$ is a characteristic scale of weak interactions ( for larger lengths our World is three-dimensional and almost orthogonal, for an empty space with the $10^{-15}$ precision); $10^{-13} \mathrm{~cm}$ is a characteristic size of proton and atomic nuclei, $10^{28} \mathrm{~cm}$ is the radius of our Metagalaxy or the observable part of our Universe.

(See: Appendix 1 - The expressions for masses of ultimate particles; Appendix 2 - A qualitative distinction between the theory of byuons and previous physical theories. See in [21] "Force-free physics. A qualitative pattern of a common approach to unifying all interactions. A novel principle of relativity.")

It should be also noted that to calculate the fundamental constants $\mathrm{h}, \mathrm{e}_{0}, \mathrm{c}$; constants of known interactions, masses of main baryons, leptons, and mesons, only three numbers $\tilde{x}_{o} \tau_{0},|\mathbf{A g}|$ should be given since the characteristic dimensions $\tilde{x}_{o} \approx 10^{-17} \mathrm{~cm}$, $\mathrm{ct}^{*} \approx 10^{-13} \mathrm{~cm}$ and $10^{28} \mathrm{~cm}$ are found from the minimum PE of byuons and from the information theorem.

\section{Dark Matter and Dark Energy in the Byuon Theory}

Determine the average density of substance in the Universe while taking $i=N k P$ and, hence, its characteristic dimension $\tilde{x}_{0} N k P \approx 10^{28} \mathrm{~cm}$ (it coincides with the assumed radius of the Universe). The total energy in the Universe can be represented as $\frac{h}{\tau_{0}} N k P$. Its value is $5.4 \times$ $10^{77} \mathrm{erg}$, and the corresponding equivalent mass $\approx 6 \times$ $10^{56} \mathrm{~g}$. The uniformity of distribution of substance over the sphere with the radius $\tilde{x}_{0} N k P$ gives the density of substance in the Universe $\approx 10^{-29} \mathrm{~g} \mathrm{~cm}^{-3}$, which is measured in the known observations.

Through the set $\{A\}$, information exchange occurs between points of the set $\{D\}$, which is the main mechanism determining physical essence of the Heisenberg uncertainty interval in the conception of physical space and physical vacuum. Without introducing the points $\{A\}$, the connection between $D$-points is realized in one direction only, in that of increasing index $i$ with the speed no greater than $\mathrm{c}_{0}$.

According to the developed conception of physical vacuum structure, we can determine a momentum and a coordinate of such a complex object as an elementary particle only with an accuracy of the momentum and the coordinate entering into the relationship $P_{D}{ }^{i+1} Y_{A D}{ }^{i, i+1}=h$ governing the momentum of $D$-points mapped into $R_{3}$ 
according to (22). Here $\mathrm{Y}_{\mathrm{AD}}^{\mathrm{i}, \mathrm{i}+1}$ is any distance in one-dimensional space between the points A and D (cf. Figure 7). That is, writing the uncertainty relation in $R_{3}$ for some elementary object $\gamma$ as $\Delta P \cdot \Delta X \geq h / 2$, we mean $\Delta P$ and $\Delta X$ to be caused by the process of $R_{3}$ formation from byuons, i.e. determined by quantities of $P_{D}$ and $Y_{A D}$ type.

Its momentum corresponding to the minimum momentum for elementary particles, can be given in general form as [20-22]

$$
P=\Phi \cdot E_{k_{\min }}^{0} / c
$$

where $\Phi$ is probability of observing the object $4 \mathrm{~b}$ formed in the process of the four-contact interaction in some region of space $R_{3}$.

If the objects $4 \mathrm{~b}$ are free (that is, they create not an elementary particle but space free of them), then $\Phi=\frac{1}{16} \cdot \frac{\tilde{x}_{0}^{3}}{4 \pi x_{0}^{2} \tilde{x}_{0}}$, where $\tilde{x}_{0} \approx 10^{-33} \mathrm{~cm}$, and $x_{0} \approx 10^{-17} \mathrm{~cm}$. In this case, if the scatter in values of the momentum is $\mathrm{P}$ for an elementary object $\Delta \mathrm{p}$, then the uncertainty in the coordinate in $R_{3}$ for the object $4 \mathrm{~b}$ will be equal to $10^{28} \mathrm{~cm}$. This value $\Delta X$ has given us earlier the possibility to obtain the density of matter in the Universe, observed in experiment, by way of averaging it over the sphere $10^{28} \mathrm{~cm}$ in radius. From the modern point of view [cf. Arxiv: 0710.3018v1 [physics.gen-ph] 16 Oct.2007 ] the $4 \mathrm{~b}$-objects with $\mathrm{m}_{4 \mathrm{~b}} \mathrm{c}^{2}=33 \mathrm{eV}$ and $\Delta X=10^{28} \mathrm{~cm}$, form the so-called the cold dark matter - the quantum medium corresponding to the observed physical $R_{3}-$ space

If the object $4 \mathrm{~b}$ is not free (that is, it forms the internal geometry of an electron, for instance), then

$$
\Phi=\frac{1}{16} \cdot \frac{x_{0}^{3}}{4 \pi\left(N x_{0}\right)^{2} x_{0}},
$$

and we can write the following expression for an assemblage of objects $4 \mathrm{~b}$ forming an electron (for which $m_{e} c^{2}=$ $\left.\mathrm{N} \cdot \mathrm{E}_{k \min }\right)$ :

$$
\Delta p=\frac{1}{16} \cdot \frac{x_{0}^{3}}{4 \pi\left(N x_{0}\right)^{2} x_{0}} \cdot \frac{N E_{k_{\min }}^{0}}{c}=\frac{1}{64 \pi} \cdot \frac{E_{k_{\min }}^{0}}{N c}
$$

Using Equation (24) we obtain the uncertainty in coordinate $\Delta x$ in $R_{3}$ of the order to $10 \mathrm{~cm}$ for the assemblage of $\mathrm{N}$ objects $4 \mathrm{~b}$, that is, the electron, due to wave properties of $\mathrm{N}$ objects $4 \mathrm{~b}$, carries information on its properties not over distances of $10^{-8} \mathrm{~cm}$ (characteristical dimension of the de Broglie wave for electron at the temperature of $300 \mathrm{~K}$ ) as would be in the case of a pointwise particle but over distances of the order to $10 \mathrm{~cm}$.

When considering not $\mathrm{N}$ objects but one object $4 \mathrm{~b}$ in the electron (that is, when Formula (23) is valid) then $\Delta x$ $\approx 10^{5} \mathrm{~cm}$. Hence the less is an information on conditions of internal spatial characteristics of electron, the more is the scatter in coordinate.
In the modern terminology [cf. Arxiv: 0710.3018v1 [physics.gen-ph] 16 Oct.2007 ] 4b-objects forming elementary particles (their charges, masses and so on) and having $\Delta X$ from 10 to $10^{5} \mathrm{~cm}$ create the hot or warm dark matter.

In [21] a qualitative pattern of a common approach to unifying all interactions is shown

The byuon theory predicts a new anisotropic interaction of natural objects with physical vacuum.

Peculiar "taps" to gain new energy are elementary particles because their masses are proportional to the modulus of some summary potential $\mathbf{A}_{\Sigma}$ that contains potentials of all known fields (Appendix 1). The value of $\mathbf{A}_{\Sigma}$ cannot be larger than the modulus of $\mathbf{A}_{\mathrm{g}}$ [20-22]. In accordance with the experimental results shown in $[22,31]$, this force ejects any substance from the area of the weakened $\mathbf{A}_{\Sigma}$ potential along conal surfaces at angles of $100^{\circ} \pm 10^{\circ}$ around the vector $\mathbf{A}_{\Sigma}$ direction. This vector has the following coordinates in the second equatorial system of coordinates: right ascension $\alpha \approx 293^{\circ} \pm 10^{\circ}$ $\left(19^{\mathrm{h}} 20^{\mathrm{m}}\right)$, declination $\delta \approx 36^{\circ} \pm 10^{\circ}[22,31]$. The vector $\mathbf{A}_{\Sigma}$ is parallel to the vector $\mathrm{A}_{\mathrm{g}}$ practically.

The new force is of nonlinear and non-local character as to variation of some summary potential $\mathbf{A}_{\Sigma}$ and may be represented by some series in $\Delta \mathbf{A}_{\Sigma}$ [20-22,32].

The expression for the new force takes the form :

$$
|\vec{F}|=-2 N m_{0}\left|\vec{A}_{\mathrm{G}}\right| \cdot c^{2} \lambda(\Delta A) \cdot \frac{\partial \lambda(\Delta A)}{\partial \Delta A} \cdot \frac{\partial \Delta A}{\partial X_{1}}
$$

Here $N$ is the number of stable elementary particles in the body (electrons, protons and neutrons).

Note that expression for the new force (25) is local (we cannot deal with the nonlocal ones as yet), therefore, to account for the nonlocality of the phenomenon, we will take $\Delta A$ equal to the difference in changes of the summary potential $\left|\vec{A}_{\Sigma}\right|$ at the location points of a test body and a sensor element [20-22].

These changes being equal, the force will be absent. Depending on the relative position of the sensor and the test body, $\Delta A$ can take as a positive, so a negative value.

To estimate a role of gravitational field in a change of $\left|\vec{A}_{\Sigma}\right|$ we put forward the maximal gravitational potential $\varphi_{\max }$, determined for proton by the following relationship:

$$
\mathrm{m}_{\mathrm{p}} \varphi_{\max }=\mathrm{e}\left|\mathrm{A}_{\mathrm{g}}\right|,
$$

where $\mathrm{m}_{\mathrm{p}}$ is the proton mass. Then the contribution of $\varphi_{\max }$ in the change of $\left|\vec{A}_{\Sigma}\right|$ is described by the following equality:

$$
\varphi_{\max } \cos \mathrm{m}_{\mathrm{p}}=\mathrm{eAv} / \mathrm{c},
$$

where $\mathrm{v}$ is in this case the velocity of our Galaxy relative to the neighbouring galaxies, $\cos =\cos _{I I^{-} I I^{-}}^{N k P-i-2, N k P-i}$ (15). It characterizes nonorthogonality of our World at the moment of the formation of the space of elementary par- 
ticles.. It is worth noting that potentials of physical fields have the physical meaning only for interacting byuons when elementary particles are generated with their masses and charge numbers. As for the vector potential of magnetic field it is gauged so that its value on the axis for example of the solenoid equals zero; $x_{1}$ is the coordinate, directed from the point of the most decreased $\vec{A}_{\Sigma}$ on a winding to the vector $\vec{A}_{G}$.

The analysis of the specific experimental results with high field magnets (see [20-22,32-35]) has led to the following expression for $\lambda(\Delta A)$ :

$$
\lambda(\Delta A)=\sum_{k=1}^{\infty} \lambda_{k} \exp \left\{-\left[\frac{\Delta A}{A_{\mathrm{G}}} \cdot \frac{r}{\Delta y} \cdot\left(\frac{c t^{*}}{x_{0}}\right)^{3 / 2}\right]^{k}\right\} \cdot \Delta A^{k}
$$

Here $r$ is the radius of the circle where the test body is located on; $\Delta y$ is the difference in coordinates $y$ of the sensor and the body [20-22]; $\left(x_{0} / c t^{*}\right)^{3 / 2}$ is the part of energy $2 m_{v_{e}} c_{0}^{2}=2 m_{0}\left|\vec{A}_{\mathrm{G}}\right| c_{0}^{2}$, which can be acted upon by the electromagnetic field potentials.

Using the linear term only in the expansion of (25) by $\Delta \mathrm{A}$, we obtain the following formula for the modulus of the new force:

$$
\mathrm{F}=2 \mathrm{Nm}_{v} \mathrm{c}^{2} \lambda_{l}^{2 \cdot} \Delta \mathrm{A}_{\Sigma}\left(\Delta \mathrm{A}_{\Sigma} / \Delta \mathrm{X}\right)
$$

It is worth noting that the experiments for the scanning the celestial sphere by the pulsed plasma generator [22,31] to detect some directions in space, where energy is more than the average value, are the final stage in the determination of the direction of the new force. This direction was determined before by the using the high field magnets [20-22,32-35], by the investigations of the rate of $\beta$ decay for a number of radioactive elements [20-22,24, $25,36]$, by the investigations with high precision gravimeters [20-22,37], and by plasma generators of other types $[20-22,38]$.

Experiments with high field magnets $[1,2]$ showed that the new interaction had the most probably an isotropic component as well.

Let us discuss the nature of the dark energy in the framework of the byuon theory on the base of potentials of physical fields.

It is known that the gravitational potential $\varphi$ is negative, and therefore for any summation of potentials it decreases the modulus of $\mathbf{A}_{\Sigma}$. Masses of elementary particles are proportional to this modulus. Hence the new force will push out any material body from the region of the decreased modulus of $\mathbf{A}_{\Sigma}$, because a defect of energy $\Delta \mathrm{E}=\Delta \mathrm{mc}^{2}$ will appear and the corresponding force will act to the region with undisturbed value of $\mathbf{A}_{\Sigma}$. Any material body decreases in its own region the modulus $\mathbf{A}_{\Sigma}$. due to potentials of physical fields of all its elementary components, i.e. creates the gradient $\Delta \mathrm{A}_{\Sigma} / \Delta \mathrm{X}$. Gravitationally acting mass, for example, our Galaxy, creates around itself the gravitational potential $\varphi$. To estimate the action of one galaxy to another we put in the Formula (29) the potential $\varphi_{\max }$ from (26) and $\Delta \mathrm{A}_{\Sigma}$ from (27) $\left(\Delta \mathrm{A}_{\Sigma}=\mathrm{A}\right)$. Let us estimate the distance $\mathrm{R}_{\mathrm{GG}}$ where the new force $\mathrm{F}$ from (29) will be higher than the gravitational force $\mathrm{F}_{\mathrm{g}}$ (Figure 1):

$$
\mathrm{R}_{\mathrm{GG}} \geq \mathrm{GM}_{\mathrm{g}}{ }^{2} /\left(2 \mathrm{Nm}_{\mathrm{v}} \mathrm{c}^{2} \lambda_{\mathrm{l}}{ }^{2} \cos ^{2} \varphi_{\max }{ }^{2}\left(\mathrm{~m}_{\mathrm{p}} \mathrm{c} / \mathrm{ve}\right)^{2}\right)
$$

where $\mathrm{G}$ is the gravitational constant.

Here $\mathbf{M}_{\mathbf{g}}$ is the mass of the one of interacting galaxies. We consider an interaction of two galaxies with $10^{10}$ stars, assume that the mass of each star is of order to the solar mass $\left(\sim 10^{33} \mathrm{~g}\right)$ and a relative velocity of each galaxy $\mathrm{v}=100 \mathrm{~km} / \mathrm{sec}$ and $1000 \mathrm{~km} / \mathrm{sec}$. From our experiments $\lambda_{l}=10^{-12}[20-22,51]$. As the result we obtain from (30) $\mathrm{R}_{\mathrm{GG}} \geq 10^{26} \mathrm{~cm}$ for $\mathrm{v}=100 \mathrm{~km} / \mathrm{sec}$ and $\mathrm{R}_{\mathrm{GG}} \geq 10^{28}$ $\mathrm{cm}$ for $\mathrm{v}=1000 \mathrm{~km} / \mathrm{sec}$.

Thus we have estimated the magnitude of the distance between galaxies above which they scatter under the action of the new force. The estimate obtained seems as reasonable and indicates that the physics of byuons is perspective to explicate the nature of dark energy and dark matter.

\section{References}

[1] M. J. Rees, "Dark Matter: Introduction," Astro-Physics, Vol. 361, 2003, pp. 2427-2434.

[2] E. A. Baltz, "Dark Matter Candidates," Astro-Physics, Vol. 1, 8 December 2004.

[3] V. Rubin, W. K. Ford and N. Thonnard, Astrophysical Journal, Vol. 238, 1980, p. 471.

[4] J. Garcia-Boldino, "Cosmology and Astrophysics," Astro-Physics, Vol. 2, 2005, p. 139.

[5] R. D. Peccei and H. R. Quinn, "CP Conservation in the Presence of Pseudoparticles," Physical Review Letters, Vol. 38, 1977, p. 1440.

[6] M. S. Turner, "Windows on the Axion," Physics Reports, Vol. 197, 1990, pp. 67.

[7] P. Sikivie, "Experimental Tests of the 'Invisible' Axion," Physical Review Letters, Vol. 51, 1983, p. 1415.

[8] S. J. Asztalos, et al. "Experimental Constraints on the Axion Dark Matter Halo Density," Astrophysical Journal, Vol. 571, 2002, p. L27.

[9] H. Pagels and J. R. Primack, "Supersymmetry, Cosmology, and New Physics at Teraelectronvolt Energies," Physical Review Letters, Vol. 48, 1982, p. 223.

[10] G. Jungman, M. Kamionkowski and K. Griest, "Supersymmetric Dark Matter," Physics Reports, Vol. 267, 1996, p. 195.

[11] L. Covi, J. E. Kim and L. Roszkowski, "Axinos as Cold Dark Matter," Physical Review Letters, Vol. 82, 1999, p. 4180.

[12] A. Kusenko and P. J. Steinhardt, "Q-Ball Candidates for Self-Interacting Dark Matter," Physical Review Letters, 
Vol. 87, 2001, p. 141301.

[13] K. R. Dienes, E. Dudas and T. Gheghetta, "Grand Unification at Intermediate Mass Scales through Extra Dimensions," Nuclear Physics B, Vol. 537, 1999, p. 47.

[14] J. A. R. Cembranos, A. Dobado and A. L. Maroto, "Branon Dark Matter," High Energy Physics-Phenomenology, p. 0406076.

[15] Z. Berezhiani, D. Comelli and F. L. Villante, "The Early Mirror Universe: Inflation, Baryogenesis, Nucleosynthesis and Dark Matter," Physics Letters B, Vol. 503, 2001, p. 362.

[16] D. J. H. Chang, E. W. Kolb and A. Riotto, "Superheavy Dark Matter," Physical Review D, Vol. 59, 1999, p. b3501.

[17] K. Jedamzik, "Primordial Black Hole Formation during the QCD Epoch," Physical Review D, Vol. 55, 1997, p. 5871 .

[18] M. Trodden and S. M. Carrol, "TASI Lectures: Introduction to Cosmology," Astro-Physics/0401547.

[19] P. J. E. Peebles and B. Ratra, "The Cosmological Constant and Dark Energy," Reviews of Modern Physics, Vol. 75, 2003, p. 559.

[20] Y. A. Baurov, "Structure of Physical Space and New Method of Obtaining Energy (Theory, Experiment, Applications)," Moscow, Krechet, in Russian, 1998.

[21] Y. A. Baurov, "On the Structure of Physical Vacuum and a New Interaction in Nature (Theory, Experiment and Applications)," Nova Science, NY, 2000.

[22] Y. A. Baurov, "Global Anisotropy of Physical Space (Experimental and Theoretical Basis)," Nova Science, 2004.

[23] Y. A. Baurov, "Structure of Physical Space and Nature of Electromagnetic Field," in Coll. Work. PHOTON: Old Problems in Light of New Ideas, Nova Science, New York, 2000, pp. 259-269.

[24] Y. A. Baurov, "Structure of Physical Space and New Interaction in Nature (Theory and Experiment)," Proceedings of conference Lorentz group, CPT and Neutrinos, World Scientific, 2000, pp. 342-352.

[25] Y. A. Baurov, "Structure of Physical Space and Nature of de Broglie Waves (Theory and Experiment)," Journal Annales de Fondation de Broglie, Contemporary Electrodynamics, Vol. 27, No. 3, 2002, pp. 443-461.

[26] Y. A. Baurov, Y. N. Babaev and V. K. Ablekov, Doklady Akademii Nauk, Vol. 259, 1981, p. 1080.

[27] Y. N. Babaev and Y. A. Baurov, "Preprint P-0362 of Institute for Nuclear Research of Russian Academy of Sciences (INR RAS)," Moscow, 1984 (in Russian).

[28] V. V. Kolpachev and C. A. Nikolaev, "Preprint N 1695 of Institute for Space Researches of Russian Academy of Sciences (ISR RAS)," Moscow, in Russian, 1990.

[29] C. E. Shannon, Bell Labs Technical Journal, Vol. 27, 1948, pp. 379,623.

[30] A. I. Kholmogorov, "Information Theory and Theory of Algorithms," Moscow, Nauka, in Russian, 1987.

[31] Y. A. Baurov, I. B. Timofeev, V. A. Chernikov, S. F.
Chalkin and A. A. Konradov, "Experimental Investigation of the Distribution of Pulsed-Plasma-Generator at its Various Spatial Orientation and Global Anisotropy of Space," Physics Letters A, Vol. 311, 2003, p. 512.

[32] Y. A. Baurov, "Space Magnetic Anisotropy and a New Interaction in Nature," Physics Letters A, Vol. 181, 1993, p. 283.

[33] Y. A. Baurov, E. Y. Klimenko and S. I. Novikov, "Experimental Observation of Space Magnetic Anisotropy", Doklady Akademy Nauk SSSR (DAN), Vol. 315, 1990, p. 1116.

[34] Y. A. Baurov, E. Y. Klimenko and S. I. Novikov, "Experimental Observation of Space Magnetic Anisotropy," Physics Letters A, Vol. 162, 1992, p. 32.

[35] Y. A. Baurov and P. M. Ryabov, "Experimental Investigations of Magnetic Anisotropy Using Quartz Piezoresonance Balances," Doklady Akademy Nauk SSSR (DAN), Vol. 326, 1992, p. 73.

[36] Y. A. Baurov, A. A. Konradov, E. A. Kuznetsov, V. F. Kushniruk, Y. B. Ryabov, A. P. Senkevich, Y. G. Sobolev and S. Zadorozsny, "Experimental Investigations of Changes in $\beta$-Decay Rate of ${ }^{60} \mathrm{Co}$ and ${ }^{137} \mathrm{Cs}$," Modern Physics Letters A, Vol. 16, No. 32, 2001, p. 2089.

[37] Y. A. Baurov and A. V. Kopaev, "Experimental Investigation of Signals of a New Nature with the Aid of Two High Precision Stationary Quartz Gravimeters," Hadronic Journal, Vol. 25, 2002, p. 697.

[38] Y. A. Baurov, G. A. Beda, I. P. Danilenko and V. P. Ignatko, "Experimental Investigation of New Method of Energy Generation in Plasma Devices Caused by Existence of Physical Space Global Anisotropy," Hadronic Journal Supplement, Vo. 15, 2000, pp. 195-210.

[39] R. A. Alpher, M. Bethe and G. Gamov, Physical Review, Vol. 70, 1946, p. 572; Vol. 73, 1948, p. 803.

[40] A. D. Linde, Uspehi Fizicheskih Nauk, Vol. 144, 1984, p. 177.

[41] G. Y. Bogoslovsky, "Theory of Locally Anisotropic Space-Time," edited by Moscow State University, in Russian, 1992.

[42] K. Rund, "Differential Geometry of Finsler's Spaces," Moscow, Nauka, 1981.

[43] "Time Structures in Natural Sciences: On the Road to Right Understanding of Time Phenomenon," Part 1, Interdisciplinary Research, edited By Moscow State University, in Russian, 1996.

[44] N. N. Bogolyubov and D. V. Shirkov, "Introduction to the Theory of Quantized Fields," Moscow, Nauka, in Russian, 1976.

[45] L. B. Okun', "Leptons and Quarks," Nauka, Moscow, in Russian, 1990.

[46] J. H. Shwartz, "Superstrings," World Scientific, Singapore, 1985.

[47] L. D. Landau and E. M. Lifshitz, "Theory of the Field," Nauka, Moscow, in Russian, 1967.

[48] H. Weyl, "Raum, Zeit Material Vierte Erweiterte Auflage,” Springer, Berlin, 1921. 
[49] H. Weyl, "Raum, Zeit, Materie, FüNfte, Umgearbeitete Auflaqe," Springer, Berlin 1923.

[50] P. A. M. Dirac, Proceedings of the Royal Society, London, 1973, Vol. A333, p. 419.
[51] Y. A. Baurov, A. A. Shpitalnaya and I. F. Malov, "Global Anisotropy of Physical Space and Velocities of Pulsars," International Journal of Pure \& applied Physics, Vol. 1, No. 1, 2005, pp. 71-82. 


\section{Appendix 1:}

The Planck constant and electric charge is determined by the following Expressions [20-22]:

$$
\begin{gathered}
\mathrm{h}=\left(\left(\left[\mathrm{A}_{\mathrm{I}^{*}} \mathrm{X}_{\mathrm{O}}\right]_{\mathrm{II}}^{+} *\left[\mathrm{~A}_{\mathrm{I}^{*}} \mathrm{X}_{\mathrm{O}}\right]_{\mathrm{I}}^{-}\right) / \mathrm{c}_{\mathrm{o}}\right) * \mathrm{X}_{\mathrm{o}} / \mathrm{ct}^{*} \\
\mathrm{e}_{\mathrm{o}}^{2}=(1 /(4 \sqrt{3})) * \mathrm{~A}_{\mathrm{\Gamma}}^{2} \mathrm{x}_{\mathrm{o}}^{2}\left(\mathrm{x}_{\mathrm{o}} / \mathrm{ct}^{*}\right)^{3 / 2}
\end{gathered}
$$

The masses of ultimate particles can be described by the following Expressions [20-22]:

$$
\begin{aligned}
& m_{p} c^{2}=\frac{\sqrt{h c}}{2}\left(\frac{4 \sqrt{3} e_{0}^{2}}{h c}\right)^{3}\left(1-\frac{1}{2} \sqrt{\frac{3 x_{0}}{c t^{*}}}\right) \times \\
& {\left[\sqrt{1+\frac{1}{6\left(1-\frac{1}{2} \sqrt{\frac{3 x_{0}}{c t^{*}}}\right)^{2}}}+1\right]|\vec{A}|} \\
& m_{\pi^{0}} c^{2}=\frac{\sqrt{h c}}{2}\left(\frac{4 \sqrt{3} e_{0}^{2}}{h c}\right)^{3}\left(1-2 \sqrt{\frac{3 x_{0}}{c t^{*}}}\right) \times \\
& {\left[\sqrt{1+\frac{1}{6\left(1-2 \sqrt{\frac{3 x_{0}}{c t^{*}}}\right)^{2}}}-1|\vec{A}|\right.} \\
& =924 \mathrm{meV},
\end{aligned}
$$

Masses of all leptons:

$$
\begin{gathered}
2 m_{v_{0}} c^{2}=\frac{\sqrt{h c}}{2 k} \cdot\left(\frac{h c}{4 \sqrt{3} e_{0}^{2}}\right) \cdot|\vec{A}|, \\
m_{e} c^{2}=\frac{\sqrt{h c}}{2 k} \cdot\left(\frac{h c}{4 \sqrt{3} e_{0}^{2}}\right)^{3} \cdot|\vec{A}|, \\
2 m_{\nu_{\mu}} c^{2}=2 \cdot 3^{1 / 4} \sqrt{h c} \cdot\left(\frac{4 \sqrt{3} e_{0}^{2}}{h c}\right)^{5.5} \cdot|\vec{A}| \\
m_{\mu} c^{2}=3^{1 / 4} \sqrt{h c} \cdot\left(\frac{4 \sqrt{3} e_{0}^{2}}{h c}\right)^{3.5} \cdot|\vec{A}| \\
2 m_{v_{\tau}} c^{2}=3^{1 / 4} \cdot 96 \cdot \sqrt{2} \cdot \sqrt{h c} \cdot\left(\frac{4 \sqrt{3} e_{0}^{2}}{h c}\right)^{5.5} \cdot|\vec{A}| \\
m_{\tau} c^{2}=3^{1 / 4} \cdot 12 \cdot \sqrt{2} \cdot \sqrt{h c} \cdot\left(\frac{4 \sqrt{3} e_{0}^{2}}{h c}\right)^{3.5} \cdot|\vec{A}| \cdot
\end{gathered}
$$

\section{Appendix 2:}

It is known that any novel physical model of the Universe must meet the following criteria. First, all the dis- covered laws of nature as well as sufficiently well established models of one or another physical phenomena must follow from the new model as asymptotical approximations. Second, the new theory should have the capability for predictions. That is, it should guide an experimental way to the gain of new knowledge, as the theory itself gives nothing but only points such a way. Criterion of truth is an accurately performed experiment independently confirmed by various authors. The theory of byuons [20-22] seems to meet the above criteria. That is a theory of "life' of special discrete objects from which the surrounding space and the world of elementary particles form. The intrinsic dynamics of byuons determines such fundamental phenomena as the course of time, rotation of planets and stars, spins of elementary particles, asf.

What is a qualitative distinction between the theory of byuons and previous physical theories?

First, the physical space was always given, in one way or another, and motion equations for a system of objects under study were written in that space. Space could be uniform continuum (Newton, Minkovsky) or discrete, one-dimensional or multidimensional, asf. In present-day cosmological models of the Universe origin (the Gamov's Big Bang [39], the Linde's model of bulging Universe [40], and so on), space is always given, too. But in the theory of byuons, the physical space (necessarily three-dimensional one, not ten-or-moredimensional as in some modern physical models) is a special quantized medium arising as the result of interaction of byuon's vacuum states (VSs). That is, space is not given but arises. Therewith the appearing three-dimensional space must have an insignificant global anisotropy, as distinct from all basic isotropic models with the same properties in various directions. The said anisotropy denotes the existence of some chosen direction caused by the existence, in nature, of a new fundamental vectorial constant, the cosmological vector-potential $\mathrm{A}_{\mathrm{g}}$ entering into the definition of the byuon. That new constant is associated with the prediction of a novel anisotropic interaction of natural objects between themselves and with the physical vacuum, a lowest energy state of physical fields.

It should be noted that in the literature spaces with local rather than global anisotropy are considered [41], for example, the Finsler's space-time [42], but the local anisotropy is given therein "by hand". That is, an author himself directively introduces it into his model instead of obtaining from some general principle. For example, there are domain models of the Universe.

Secondly, the physical sense of time notion is not yet revealed in science in the present state of the art [43]. The general philosophic concept of time as a form of matter existence, which expresses the order of change of objects and phenomena as a sequence of events, does not indicate a common nature of those events. As a rule, 
people tie their time to a particular periodic process: rotation of the Earth around its axis, Earth's orbiting around the Sun, oscillations of a quartz system, asf, without becoming aware of inner, profound sense of time. Standard physical time references, for example, quantum or, what is the same, atomic clock with instrument error on the level of $10^{-11}$ per year and moderate resolution of the order to $10^{-13}$ seconds, give us no possibility of approaching the knowledge of time essence. The byuon theory reveals physical essence of time as a discrete sequence of changes in the byuon's "length", its quantum number. A possibility therewith arises, to synchronize clocks at great distances comparable with dimensions of our Metagalaxy, due to the quantum process of physical space formation from the byuon's vacuum states (VSs). That possibility distinguishes substantially the theory of byuon's from A. Einstein's special theory of relativity (STR), in which clocks can be synchronized only when a signal has passed between them with speed of light $\mathrm{c}_{\mathrm{o}}$. It should be noted at once that in the byuon theory, material objects cannot move with a speed faster than the light speed (that is similar to the STR's postulate on finite propagation velocity of interactions), but synchronization of clocks occurs by a quantum way without introducing the concept of speed. That is, some object originated in the course of interaction between byuon vacuum states and forming the physical space, is at a time in two spatial regions being very distant from each other in the three-dimensional space arising.

Third, an essential distinction of the byuon theory from modern models in the classical and quantum field theories [44] is that the potentials of physical fields (gravitational, electromagnetic, asf.) become, in the theory of byuons, exactly fixable, measurable values. Recall to the reader that ordinary methods of measurement are capable to measure solely a difference of potentials. Therefore, in the existing field theory, potentials are defined only with a precision of an arbitrary constant or the rate of change of the potentials in space or time (gauge models). But in the theory of byuons, field potentials become single-valued since there are formed, on the set of byuon VSs, field charge numbers which generate the fields themselves, as, for example, the electric charge of an electron generates an electric field. The physical sense of field as a special form of matter, loses its basic meaning because all the observable events can be described on the basis of the byuon theory without introducing the concept of force, and hence of field.

An important methodic distinction between the byuon model and all those existent in the theoretical physics of today, is that the latter use images with properties of real objects, - for example, strings in the physics of elementary particles [45], superstrings, membranes when creating a unified field theory [46], asf. But the byuons are unobservable objects having no analogues in the nature though all the natural objects appear in the result of interaction of byuon VSs.

The proposed pattern of formation of the observed space $R_{3}$ on the basis of dynamics of the finite set of byuons animates, fills with a sense, and supplements the physical results on properties of elementary particles, described in $[34,35]$. For example, if some elementary object appearing in a byuon interaction has, with the probability near 1 , the vacuum state $I^{+}$of a byuon completing formation of its quantum numbers (the greatest period of byuon interaction of the order of $k N$ ), such an elementary object will be stable as well as its properties will, since quite a definite amount of information will be locked up by $\mathrm{VS} I^{+}$. This relates, for example, to the electron.

Thus, as opposed to gauge models in which the level of symmetry constantly grows for more complete, allembracing, and unified description of the surrounding world [23-28], and to obtain massive particles it is necessary to use "by hands" the Higgs mechanism (spontan violation of symmetry), in the present model there exists first a one-dimensional world (its direction, i.e. that of $\mathbf{A}_{\mathrm{g}}$, is determined by the byuon with the maximum $\mathrm{x}(\mathrm{i})$ ), then its symmetrization takes place, and the space $R_{3}$, with the world of elementary particles originates. At that some insignificant $\left(\sim 1 / \mathrm{k}\left(\sim 10^{-15}\right)\right)$ asymmetry of "empty" $R_{3}$ remain as well as that of the $10^{-5}$ order inside the elementary particles.

It should be also noted that to calculate the fundamental constants $\mathrm{h}, \mathrm{e}_{0}, \mathrm{c}$; constants of known interactions, masses of main baryons, leptons, and mesons according to formulae $(1 \div 1 \mathrm{e})$, only three numbers $\tilde{x}_{\mathrm{o}} \tau_{0},|\mathbf{A g}|$ should be given since the characteristic dimensions $\tilde{x}_{\mathrm{o}} \approx 10^{-17}$ $\mathrm{cm}, \mathrm{ct}^{*} \approx 10^{-13} \mathrm{~cm}$ and $10^{28} \mathrm{~cm}$ are found from the minimum PE of byuons and from the information theorem.

Notice that in [20-22,24,25,31-38], results of some fundamental experiments in support of the basic theoretical statements have been described 